\title{
Comparación de filtros para el análisis del espectro espacial bidimensional de Fourier, en el proceso de digitalización 3D
}

\author{
Juan Carlos Moya Morales, Jesús Carlos Pedraza Ortega, \\ Juan Manuel Ramos Arreguín, José Emilio Vargas Soto \\ Universidad Autónoma de Querétaro, Querétaro, Querétaro, México \\ moyajc@gmail.com, caryoko@yahoo.com,jsistdig@yahoo.com.mx, emilio@mecatronica.net
}

\begin{abstract}
Resumen. En el presente trabajo se muestra una comparación entre tres filtros diferentes, dentro del proceso de digitalización 3D por Transformada bidimensional de Fourier. Se lleva a cabo la implementación y comparación de un filtro por umbral, un filtro morfológico rectangular y uno elíptico, estos filtros son generados y representados mediante una máscara binaria, el cual se multiplica por el espectro espacial bidimensional de Fourier, eliminando la mayoría de las frecuencias y obteniendo una escena con franjas deformadas disminuyendo la mayor parte del ruido inicial. Para validar esta comparación, se utilizan dos objetos; un maniquí y un murciélago, se aplican los diferentes filtros a ambos objetos y se realiza la comparación de los resultados. En la etapa de filtrado también se realiza un filtro sobre la frecuencia espacial $f_{0}$, que permite obtener un patrón de franjas uniforme.
\end{abstract}

Palabras clave: Fourier bidimensional, filtro morfológico, umbral, digitalización 3D.

\section{Comparison of Filters for the Analysis of the Two-dimensional Fourier Space Spectrum in the 3D Digitization Process}

\begin{abstract}
In this paper, a comparison between three different filters in the 3D scanning processes by Fourier Transform two-dimensional. The implementation and comparison about the filter by threshold, morphological filtering like rectangle and elliptic, the representation about the filters are in a binary mask and it is multiplied by the spectrum of Fourier Transform two-dimensional, with this process the frequencies are eliminated and the new scene shows less noise than the original scene. To compeer we used two objects a manikin and a bat figure, the different filters are applied and the results are compared. In the filtering step also we filter the one pixel about $\mathrm{f}_{0}$ to get a uniform scene.
\end{abstract}

Keywords: two-dimensional Fourier, morphological filter, threshold, scanning $3 \mathrm{D}$. 


\section{Introducción}

\subsection{Perfilometria por transformada de Fourier}

La Perfilometria por Transformada de Fourier (FTP) se encuentra dentro de las técnicas de digitalización de objetos, el cual es un proceso por el cual se pueden almacenar, las características físicas de un objeto en la memoria de una computadora. La FTP ha sido objeto de análisis y estudio por muchos investigadores a lo largo de las últimas décadas, desde que sus autores Takeda, et al. lo propusieron en los 80's donde presentaron su metodología basada en la Transformada de Fourier para topografía, mostrando que era posible la identificación de valores que se encontraban en elevación y depresión de la superficie analizada, a partir de la deformación de una franja con perfil senoidal, la cual es proyectada y deformada por las características morfológicas del objeto analizado, tal como se menciona en [1]. En el siguiente año Takeda et al, [2] presentó una nueva técnica para mediciones automáticas basadas en la perfilometría por medio de la Transformada de Fourier.

A partir de estas publicaciones los trabajos presentados por muchos investigadores han tratado de mejorar el método, en el 97 Jiang Yi et al. proponen realizar cambios en la fase con lo cual analizan 4 imágenes para posteriormente realizar la obtención de la fase, siguiendo el proceso de Takeda como se menciona en [3]. La eliminación de DC, para filtrar la escena fue propuesto por Tavares, el proceso que realiza requiere de 2 escenas una con franjas deformadas verticales y otra con franjas horizontales, al obtener los espectros espaciales y sumarlos se puede identificar los límites de la componente DC para su posterior extracción, tal como lo menciona en [4]. Pedraza, et al y Abdul-Rahman presentan trabajos separados en el 2007 donde el primero realiza modificaciones mediante el análisis de discontinuidad local [5] y el segundo enfoca su tesis doctoral al análisis de franjas de Fourier y el desdoblamiento de fase [6]. En el 2008 Hussein S, et al en el 2008, analizan el tiempo de procesamiento con el algoritmo 2D-FFA y 3D-FFA que proponen.

En años recientes investigadores como Feng S, Feng L, Miguel A. Hai L, han realizado propuestas para mejorar los algoritmos de digitalización 3D por Perfilometria de Fourier, tanto del proceso general de digitalización 3D, como en [7, $8,9,10]$, así también proponen mejoras en el proceso de desdoblamiento de fase tal como se establece en $[11,12,13,14]$.

La Perfilometria por Transformada de Fourier en 2D (FTP2D) proviene de la FTP que consiste en generar y proyectar un patrón de franjas uniformes sobre una forma que será digitalizada, las franjas provocarán una escena de franjas distorsionadas, con la cual se realizara el proceso de digitalización, esta escena lleva consigo toda la información 3D del objeto.

El proceso de digitalización 3D en la FTP utiliza la configuración experimental mostrada en la figura 1, también conocida como geometría de ejes ópticos cruzados, donde los componentes básicos son un proyector, un dispositivo de captura, un equipo de cómputo para procesar los datos y un objeto a digitalizar. 


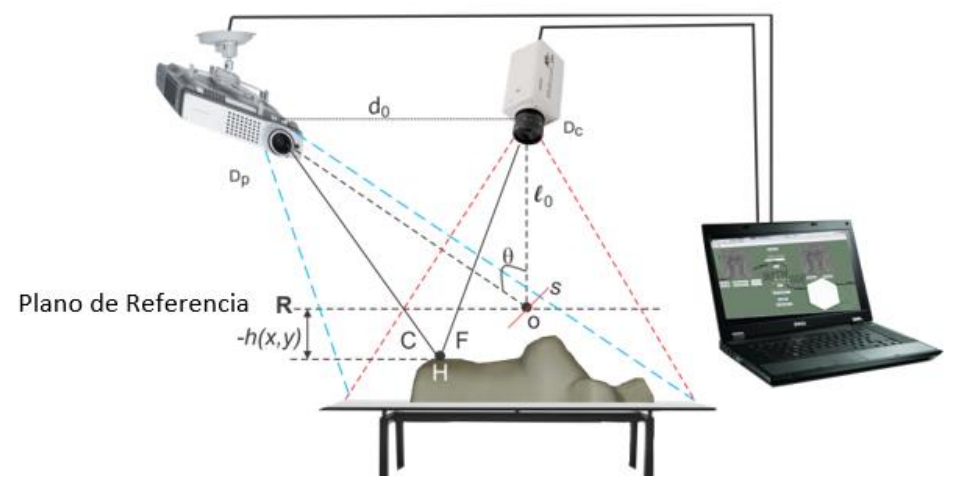

Fig. 1. Geometría de ejes ópticos cruzados usado en la FTP.

El plano de ficticio $\mathbf{R}$ mostrado en la figura 1, es utilizado de referencia para medir la altura $\mathrm{h}(\mathrm{x}, \mathrm{y})$ del objeto en particular, la lente de la cámara captura el plano deformado (escena) generado por la lente del proyector que forma su imagen conjugada en el plano R. Cuando el objeto es uniforme, la altura expresado por $\mathrm{h}(\mathrm{x}, \mathrm{y})$ es cero. La escena capturada por la cámara es expresada mediante la ecuación 1:

$$
\mathrm{g}(\mathrm{x}, \mathrm{y})=\mathrm{a}(\mathrm{x}, \mathrm{y})+\mathrm{b}(\mathrm{x}, \mathrm{y}) * \cos \left[2 * \pi \mathrm{f}_{0} \mathrm{x}+\varphi(\mathrm{x}, \mathrm{y})\right] .
$$

Acorde la ecuación $1 \mathrm{~g}(\mathrm{x}, \mathrm{y})$ representa la intensidad de la imagen en el punto $(x, y), a(x, y)$ es la iluminación del fondo, $b(x, y)$ el contraste entre las franjas claras y obscuras proyectadas, la frecuencia espacial del patrón de franjas está representada por $\mathrm{f}_{0}, \mathrm{y} \varphi(\mathrm{x}, \mathrm{y})$ corresponde a la fase que se genera por la distorsión.

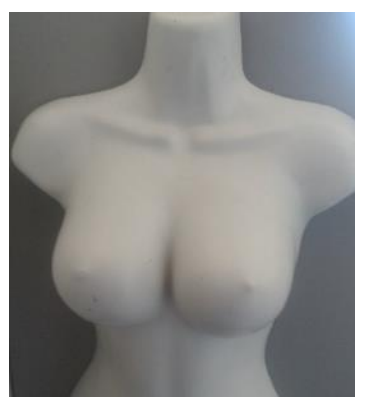

a) Maniquí

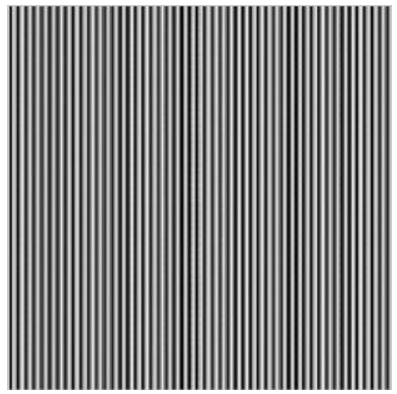

b) Patrón de franjas uniforme

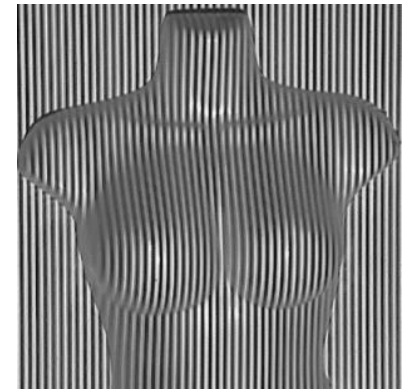

c) Patrón de franjas deformadas.

Fig. 2. Proceso de proyección de franjas.

El eje $\mathrm{x}$ es la línea que representa el plano $\mathrm{R}$ en la figura 1, siendo el eje y perpendicular a éste; Si consideramos un objeto genérico con variaciones de altura descritas por la función $\mathrm{h}(\mathrm{x}, \mathrm{y})$, el patrón de franjas capturado será un patrón de franjas distorsionado que contendrá la información 3D del objeto considerado; este 
efecto se muestra en la figura 2, donde un objeto real en este caso un maniquí se observa en la figura 2 a), el patrón de franjas uniforme que se proyectara se muestra en la figura $2 \mathrm{~b}$ ), una vez que se ha capturado la es escena se obtendrá una imagen como la mostrada en la 2 c).

La ecuación 1 también puede ser expresada como la ecuación 2.

$$
g(x, y)=a(x, y)+c(x, y) e^{\left(2 \pi i f_{0} x\right)}+c *(x, y) e^{\left(-2 \pi i f_{0} x\right)},
$$

donde

$$
c(x, y)=\frac{1}{2} b(x, y) e^{[i \varphi(x, y)]} .
$$

De la ecuación 3 el símbolo * denota el complejo conjugado.

La Transformada de Fourier en 2 Dimensiones se puede expresar como:

$$
F_{2}(u, v)=F\{f(x, y)\}=\int_{-\infty}^{\infty} \int_{-\infty}^{\infty} f(x, y) e^{-j 2 \pi(u x+v y)} d x d y
$$

Al aplicar la transformada de Fourier en dos dimensiones se obtiene una función compleja expresada por:

$$
F_{2}(u, v)=R_{2}(u, v)+i_{2}(u, v) .
$$

El espectro espacial de amplitud de Fourier se puede visualizar en forma de una imagen bidimensional, donde al centro se encuentra la frecuencia fundamental o componente de DC, los armónicos de la transformada de Fourier se visualizan posteriormente, tanto a la izquierda como a la derecha de la componente de DC, este fenómeno se puede apreciar en la figura 3 donde se observa una representación en 3D, del espectro bidimensional de Fourier, de igual forma en la figura 4 se muestra el espectro espacial en dos dimensiones desde una vista superior en escala de grises.

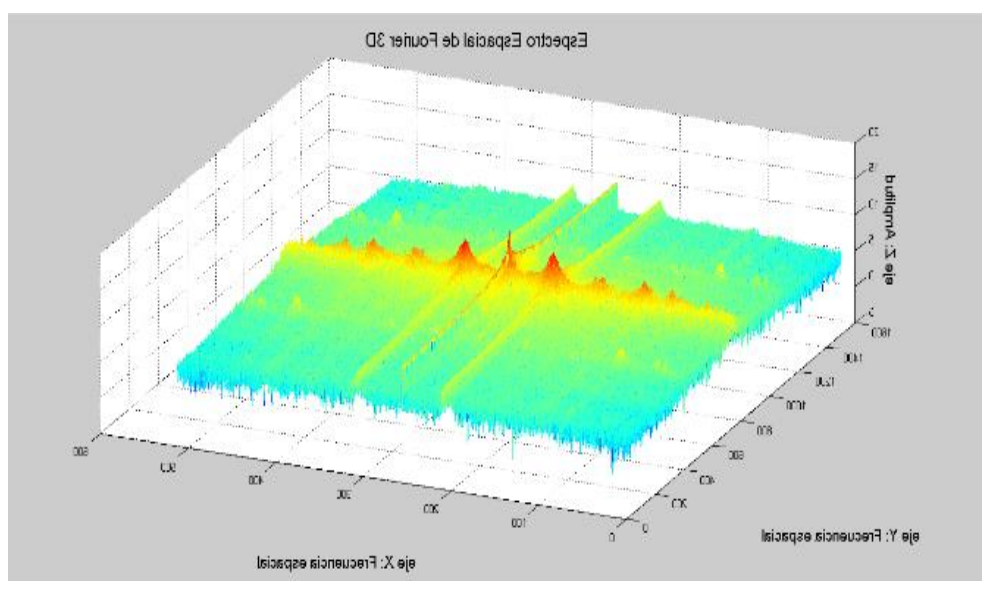

Fig. 3. Espectro espacial de Fourier vista 3D. 


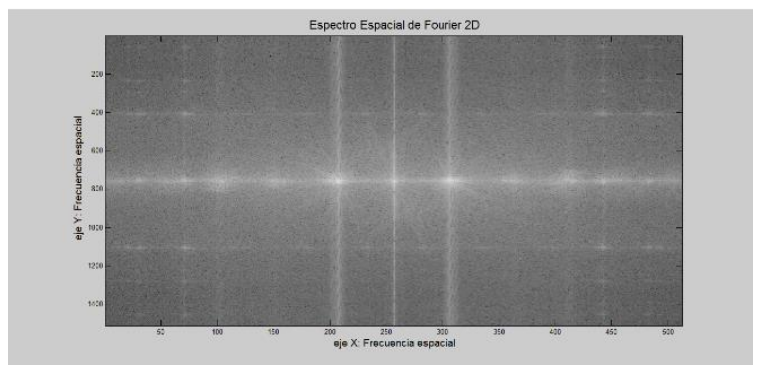

Fig. 4. Espectro espacial de Fourier vista 2D.

En este espectro se realiza el filtro $2 \mathrm{D}$ de la frecuencia espacial $\mathrm{f}_{0}$, la aplicación del filtro en esta etapa es fundamental ya que la información 3D se encuentra ubicada en esta zona. Este filtro es realizando aplicando una ventana binaria, tal como se verá más adelante. Aplicando la transformada inversa de Fourier en 2D obtenemos la función en el dominio del tiempo por lo que podemos obtener de la parte imaginaria (fase), la cual nos proporcionara los valores ortogonales, mediante la ecuación 6:

$$
\log \left[c(x, y]=\log \left[\left(\frac{1}{2}\right) b(x, y)\right]+i \varphi(x, y) .\right.
$$

De la ecuación 6 , se obtiene la fase $\varphi(\mathrm{x}, \mathrm{y})$ en la parte imaginaria, los valores obtenidos en la fase se encuentran entre $-\pi$ a $\pi$, lo cual es conocido como valores envueltos, que no permiten visualizar adecuadamente los valores $3 \mathrm{D}$ del objeto por lo que es necesario realizar un proceso denominado desdoblamiento de fase, que permita recuperar la información correctamente, la fase envuelta obtenida de la ecuación 6 se puede expresar mediante la ecuación 7 :

$$
\varphi(\mathrm{x}, \mathrm{y})=\varphi_{0}(\mathrm{x}, \mathrm{y})+\varphi_{\mathrm{z}}(\mathrm{x}, \mathrm{y})
$$

donde $\varphi_{0}(\mathrm{x}, \mathrm{y})$ es la fase producida por el ángulo de proyección correspondiente al plano de referencia, y $\varphi_{\mathrm{z}}(\mathrm{x}, \mathrm{y})$ es generada por la distribución de la altura del objeto analizado. $\varphi_{\mathrm{z}}$ es utilizada dentro de la ecuación 8 para determinar la altura $\mathrm{h}(\mathrm{x}, \mathrm{y})$ del objeto:

$$
h(x, y)=\frac{L \varphi_{z}(y)}{\varphi_{z}(x, y)-2 \pi f_{0} d}
$$

\subsection{Algoritmos de desdoblamiento de fase}

Los algoritmos de desdoblamiento de fase han sido utilizados en las últimas décadas dentro de la metodología de la Perfilometría por Transformada de Fourier, ya que los valores de las fases obtenidos se encuentran envueltos, como ya se mencionó anteriormente, por consecuencia no proporcionan las alturas correctas. Acorde a lo anterior los algoritmos de desdoblamiento de fase tratan de encontrar los valores finales, que representarían al objeto $3 \mathrm{D}$, sin embargo no son objeto de análisis del presente trabajo. 


\section{Desarrollo}

\subsection{Filtros}

Los filtros en la frecuencia espacial de Fourier y particularmente en la frecuencia espacial $\mathrm{f}_{0}$ es muy importante dentro del proceso de digitalización $3 \mathrm{D}$ ya que en esta zona se encuentra la información 3D del objeto, así mismo el utilizar un filtro de dimensiones pequeñas o muy grandes puede provocar que se procesen datos basura, que no corresponden con la información del objeto analizado, por ello se proponen la aplicación de los filtros incluidos en este trabajo.
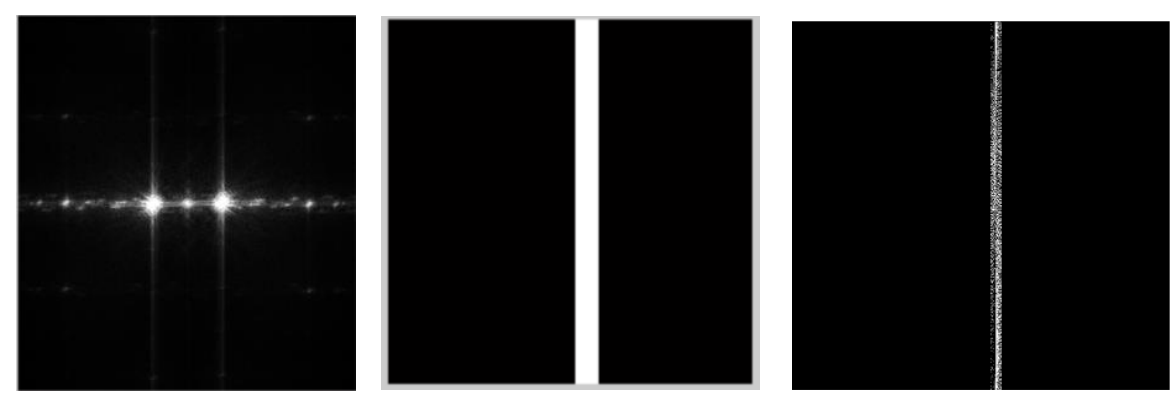

Fig. 5. Filtrado del espectro bidimensional de Fourier con el Filtro Rectangular.

Para la generación de los filtros se emplean, una matriz de filtro de las mismas dimensiones que la imagen del espectro de Fourier, de tal manera que se ubica la frecuencia espacial $\mathrm{f}_{0}$, para aplicar sobre este punto una multiplicación de matrices, filtrando de esta forma la información adecuada; Un ejemplo de filtro rectangular se puede observar en la figura 5.

Cabe destacar que el ancho de los filtros corresponde exactamente al número de franjas proyectadas sobre el objeto, ubicando el valor de la $\mathrm{f}_{0}$ en el centro del filtro.

El filtro elíptico se logra utilizando una máscara binaria de forma elíptica aplicada al espectro de Fourier, donde el centro de la elipse se encuentra en la posición de la frecuencia espacial $\mathrm{f}_{0}$, el eje menor de la elipse está determinada por el número de franjas y el eje mayor tiene sus vértices en el renglón 1 y el ultimo renglón de la imagen analizada. La figura 6 muestra un ejemplo de aplicación del filtro elíptico.
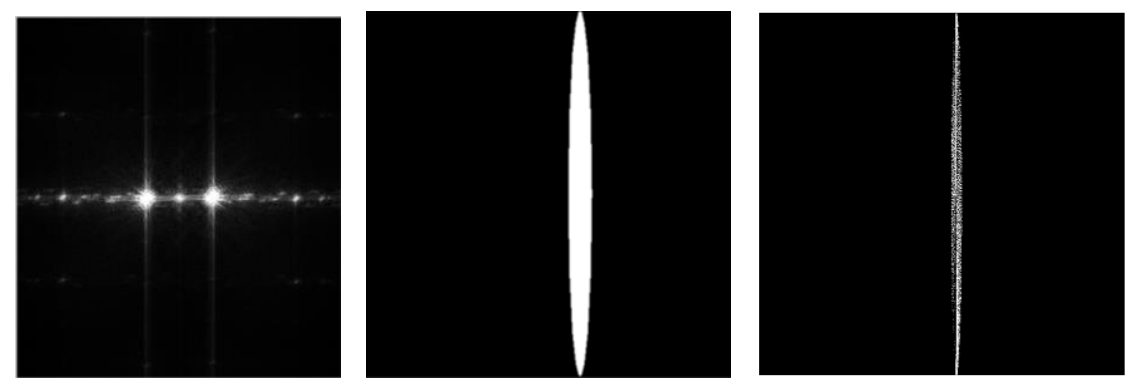

Fig. 6. Filtrado del espectro bidimensional de Fourier con el Filtro Elíptico. 
Comparación de filtros para el análisis del espectro espacial bidimensional de Fourier...

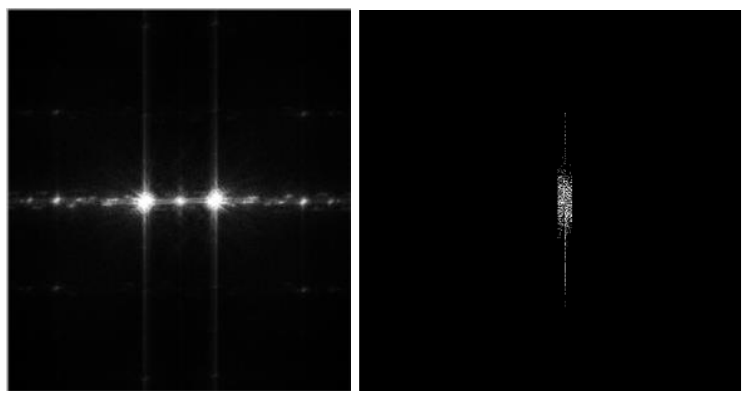

Fig. 7. Filtrado del espectro bidimensional de Fourier utilizando filtro por umbral.

El filtro por umbral se genera utilizando los márgenes del filtro rectangular, adicionalmente a este proceso se establece un valor de umbral para su filtrado, el cual está representado por la ecuación 9, donde se considera el valor de la frecuencia espacial $\mathrm{f}_{0}$ asi como el número de franjas proyectadas $\mathrm{nf}$, a partir de estos datos se genera una máscara binaria, que será utilizada como filtro al multiplicarse por el espectro espacial de Fourier 2D. El resultado de aplicar el filtro por umbral se aprecia en la figura 7:

$$
\text { Umbral }=\frac{\text { fo }}{\mathrm{nf}^{2}}
$$

\subsection{Algoritmo}

El algoritmo utilizado, para la digitalización de objetos utilizando Transformada de Fourier Bidimensional y la aplicación de los filtros propuestos se muestra en el Algoritmo 1, los requisitos para utilizar el algoritmo es una escena de franjas deformadas, la cual puede ser obtenida desde un archivo almacenado en la memoria o capturada por medio de un dispositivo de captura, como una webcam o cualquier dispositivo de adquisición de imágenes.

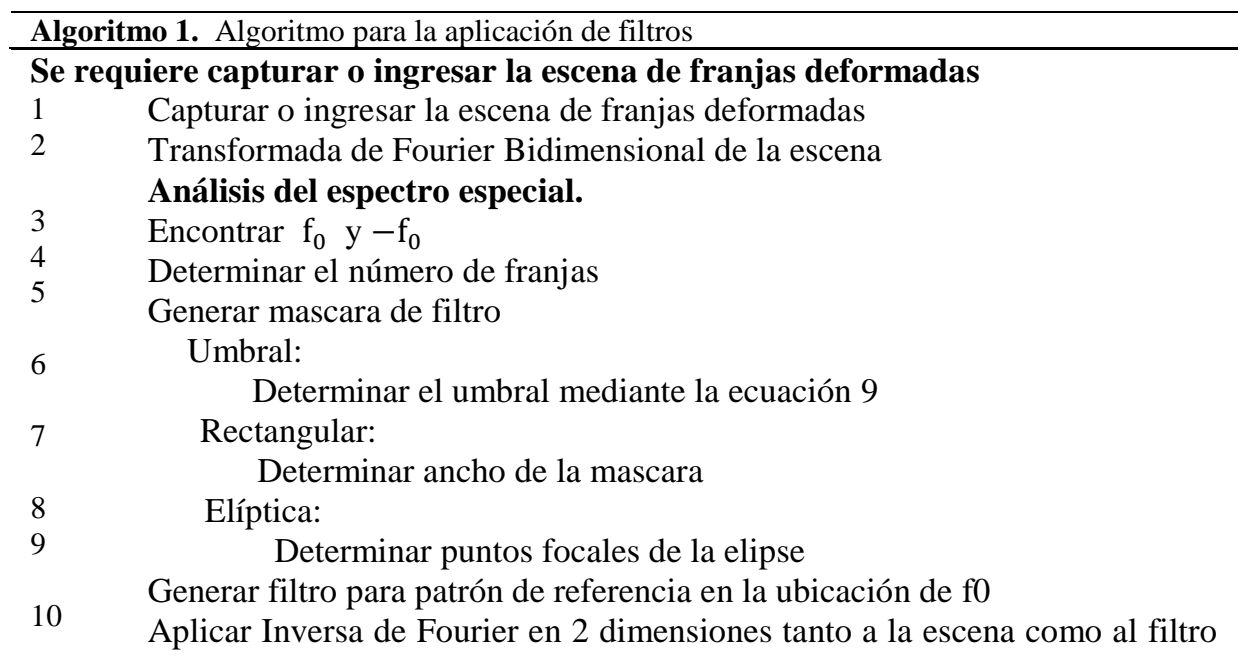


11 para el patrón de referencia.

12 Determinar el ángulo de fase en ambas imágenes.

13 Encontrar diferencia de Fases.

14 Desdoblamiento de fase sencillo.

15 Desdoblamiento de fase Graph Cut.

Mostar resultado

\section{Metodología}

La metodología propuesta se observa en la figura 8 donde como primer etapa se requiere de la proyección de las franjas sobre el objeto, el cual es capturado y se almacena la escena de la figura con las franjas deformadas, una vez que se tiene la escena en la memoria, se aplica la transformada de Fourier 2D, de este proceso se obtiene el espectro espacial de Fourier en dos dimensiones y se procede a la etapa de análisis, donde se determina el número de franjas que presenta la escena, se aplica el filtro al espectro y así mismo se adquiere la posición de la frecuencia espacial $\mathrm{f}_{0}$ y se genera una imagen de igual dimensión a la escena original.

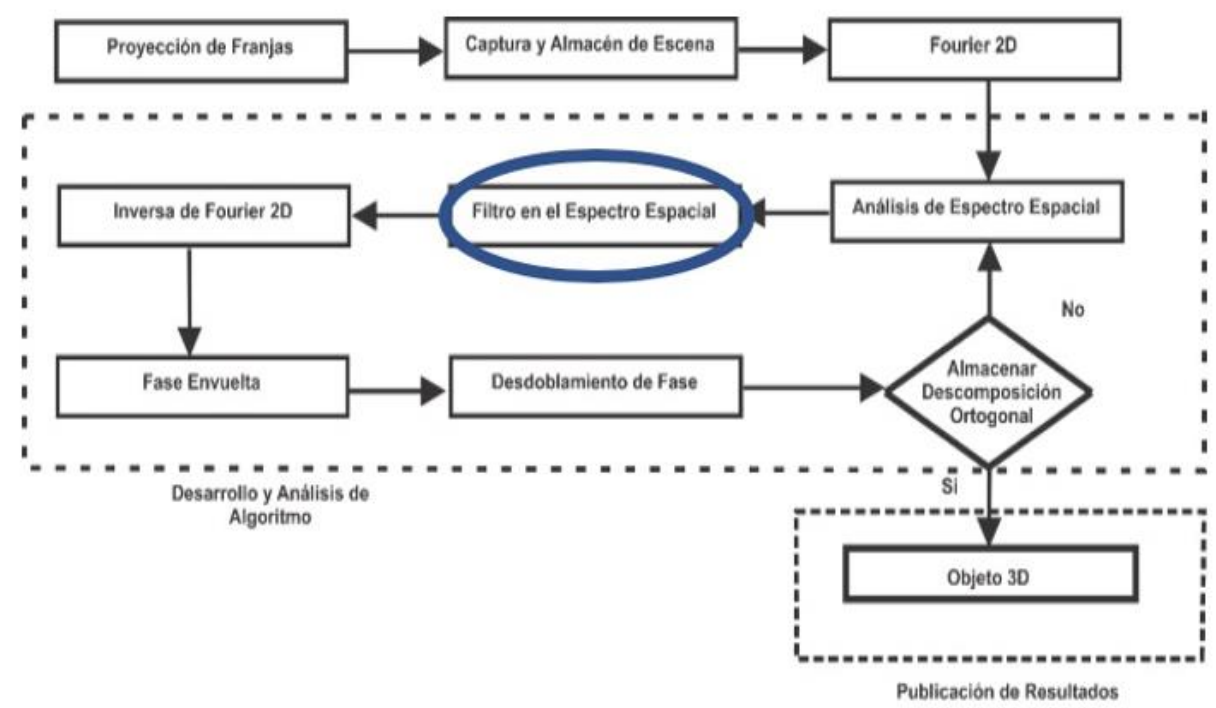

Fig. 8. Metodología propuesta.

En este trabajo se utiliza el filtro rectangular, elíptico y un filtro a base de un umbral, ya que es fundamental la aplicación de un filtro para encontrar en esta zona la información 3D del objeto. una vez que el filtro ha sido aplicado se procede a utilizar la Transformada Inversa de Fourier 2D, a ambas imágenes con lo que se obtendrá, un patrón de referencia uniforme y un patrón de franjas deformadas filtrado, posteriormente se obtiene la fase envuelta de ambas escenas y se aplica una diferencia de fases, posteriormente se utiliza el algoritmo de desdoblamiento de fase para 
verificar si el resultado es satisfactorio, ya que se mostrara la descomposición ortogonal en el espacio del objeto, de ser un resultado satisfactorio se muestra el resultado en $3 \mathrm{D}$ y de lo contrario se procede a realizar un nuevo análisis del espectro espacial de Fourier.

\section{Pruebas y resultados}

En la figura 9 se muestra la escena de franjas deformadas por la forma de un maniquí, el cual es sometido al filtro por umbral, rectangular, elíptico. En la figura 10 se muestra la aplicación de estos filtros; en 10 a) se observa el espectro espacial y la zona donde será aplicado cada uno de los filtros, en 10 b) se muestra el resultado del filtro aplicado a la izquierda se muestra el filtro por umbral, al centro el rectangular y a la derecha el elíptico; En 10 c) se muestra el resultado de la inversa de la transformada de Fourier, en 10 d) se observa una vista del objeto en dos dimensiones y en 10 e) se muestra el resultado del objeto en 3D.

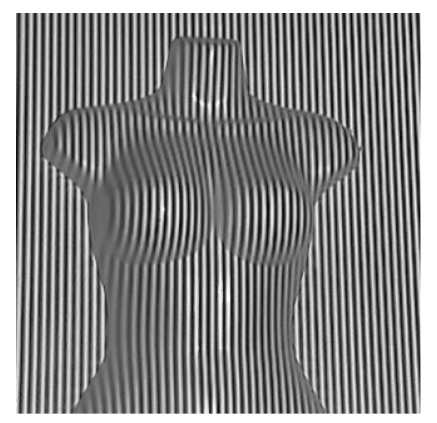

Fig. 9. Escena analizada.
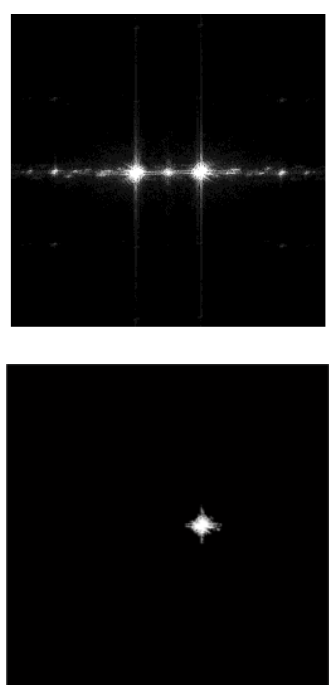

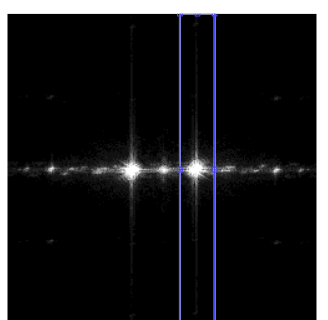

a) Espectro 2D

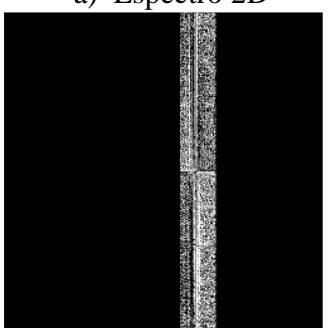

b) Filtros (Umbral, Rectangular y elíptico)
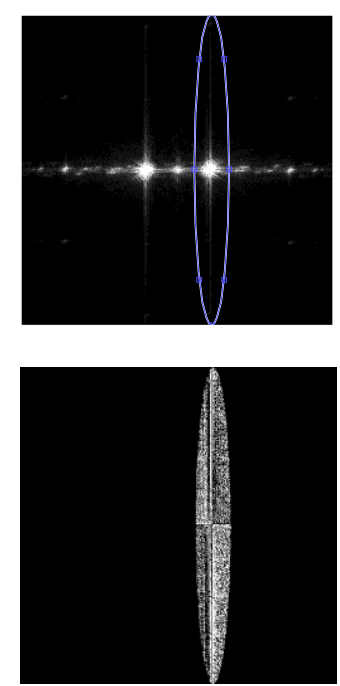
Juan Carlos Moya Morales, Jesús Carlos Pedraza Ortega, Juan Manuel Ramos Arreguín, et al.
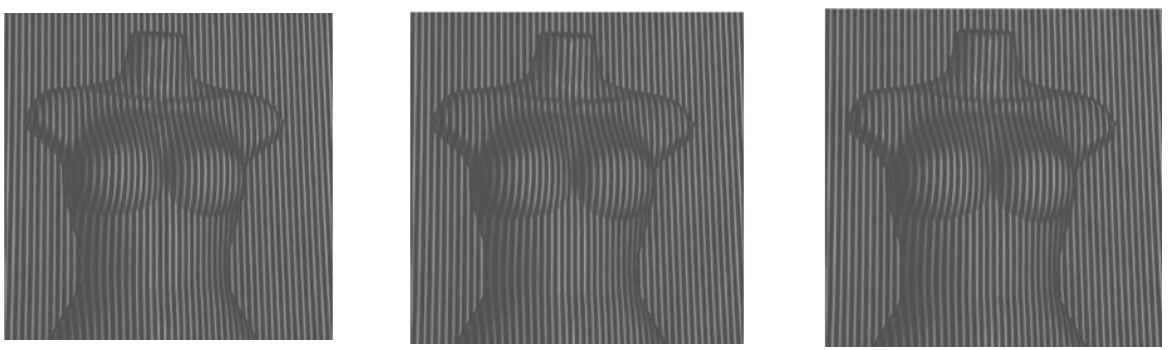

c) Resultado de la IFFT 2D
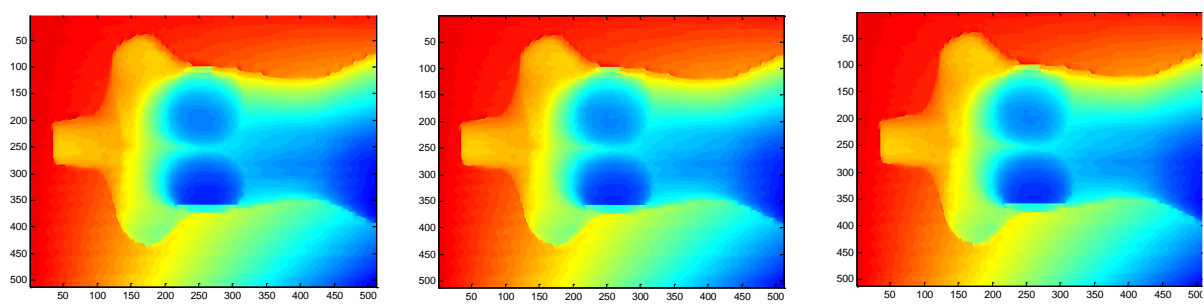

d) Vista 2D de la digitalización

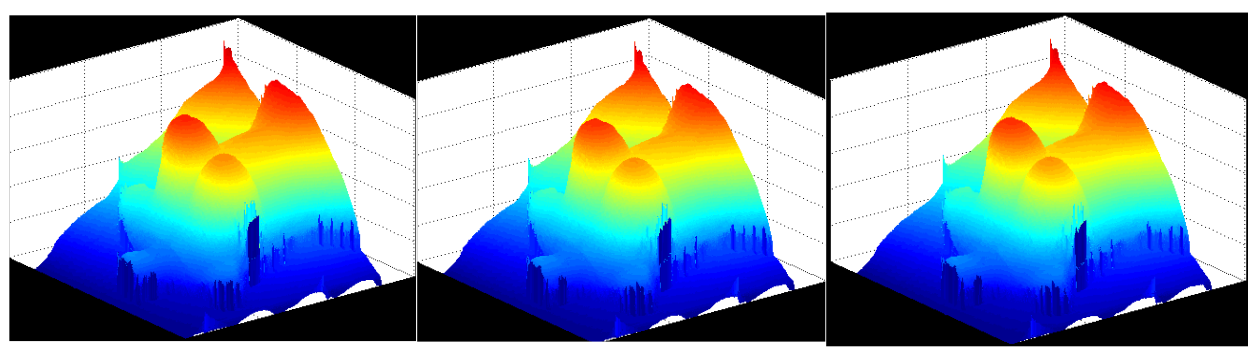

e) Objeto $3 \mathrm{D}$

Fig. 10. Digitalización de maniquí utilizando los filtros.

En la figura 11 se muestra la escena de franjas deformadas por la forma de un murciélago, el cual es sometido al filtro por umbral, rectangular, elíptico.

En la figura 12 se muestra la aplicación de estos filtros; en 12 a) se observa el espectro espacial y la zona donde será aplicado cada uno de los filtros, en 12 b) se muestra el resultado del filtro aplicado a la izquierda se muestra el filtro por umbral, al centro el rectangular y a la derecha el elíptico.

En 12 c) se muestra el resultado de la inversa de la transformada de Fourier, en 12 d) se observa una vista del objeto en dos dimensiones y en 12 e) se muestra el resultado del objeto en 3D. 
Comparación de filtros para el análisis del espectro espacial bidimensional de Fourier...

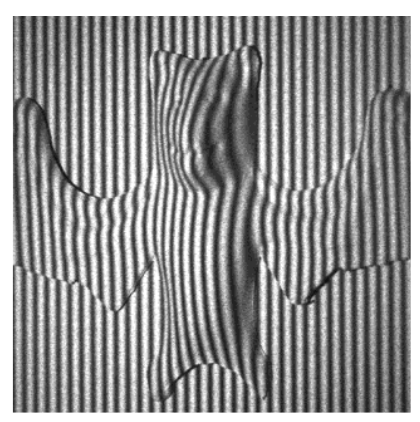

Fig. 11. Escena murcielago.
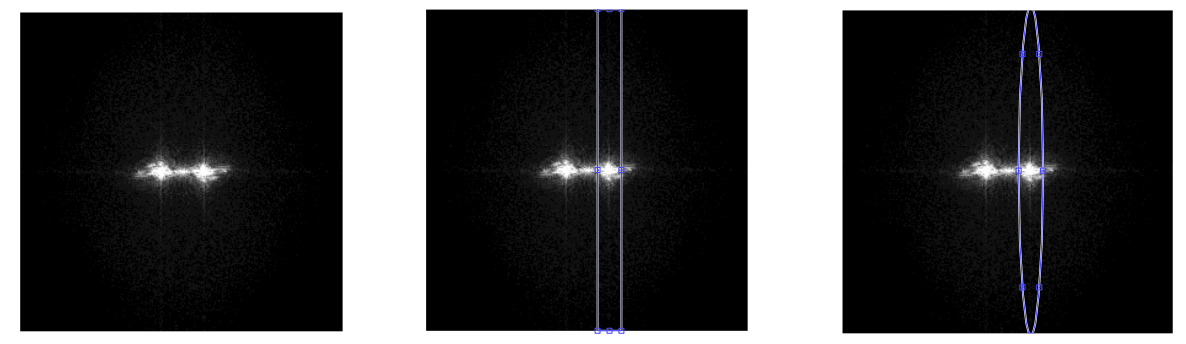

a) Espectro 2D
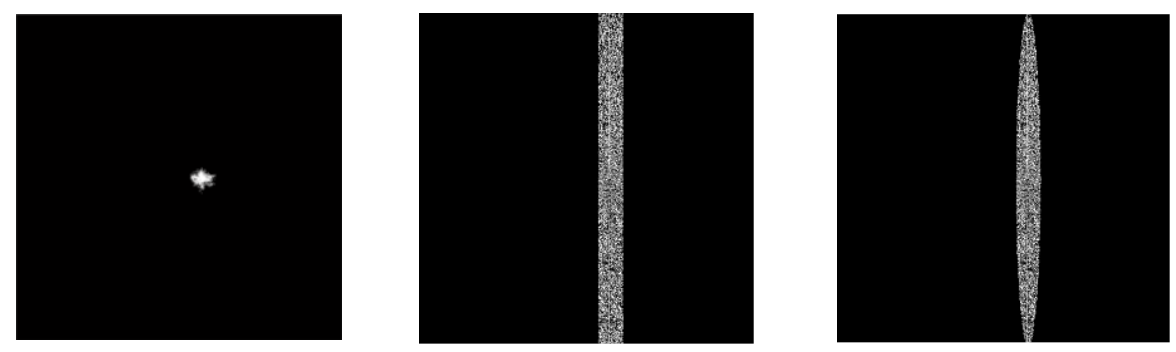

b) Filtros (Umbral, Rectangular y elíptico)
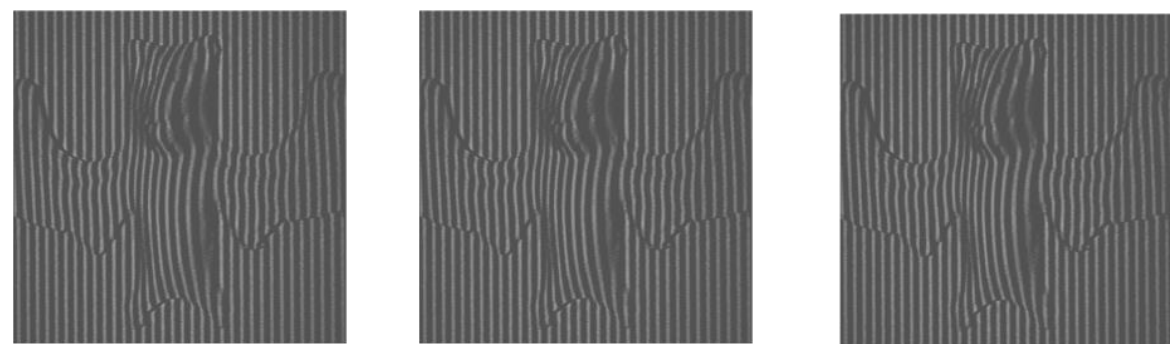

c) IFFT 2D 

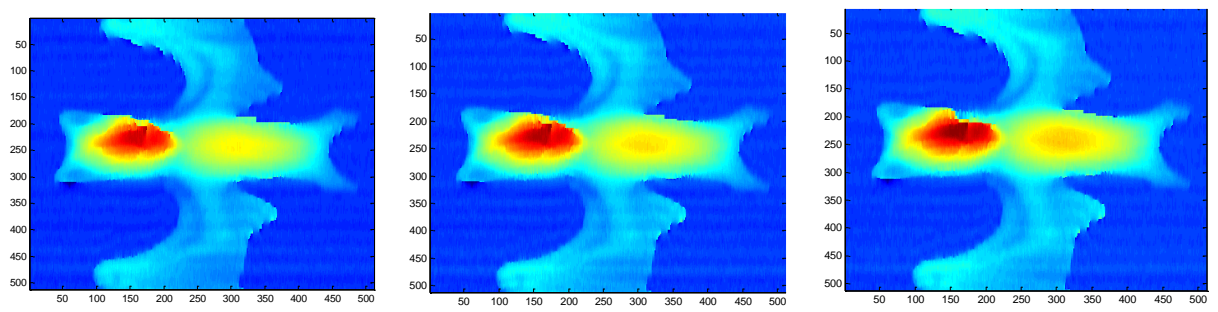

d) Vista 2D de la digitalización

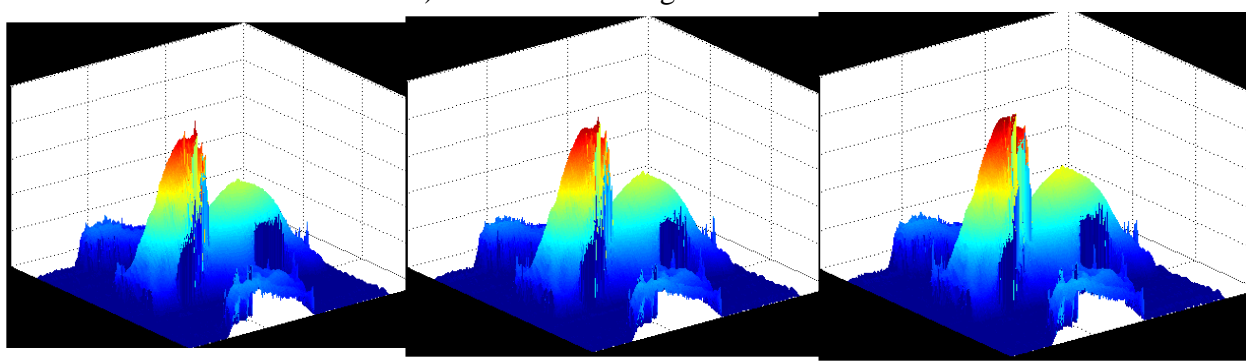

e) Objeto 3D

Fig. 12. Digitalización de murciélago utilizando los filtros.

\section{Conclusiones}

Se presentó un comparativo de tres filtros, donde los tres al terminar el proceso de digitalización 3D presentan buenos resultados ya que se aprecia correctamente las figuras a digitalizadas, aunque presentan algunas inconsistencias mínimas en ciertas zonas del objeto tales como en los bordes de los objetos y en las zonas centrales, en la zona del rostro del murciélago.

El filtro por umbral presenta la menor cantidad de inconsistencias, y valores más uniformes, el filtro rectangular muestra más inconsistencias que los resultados obtenidos con el filtro por umbral pero menos que los obtenidos por el filtro elíptico, los resultados obtenidos con el filtro elíptico presentan la mayor cantidad de inconsistencias y con valores más dispersos, se aplicó la metodología y los diferentes filtros a 10 objetos obteniendo resultados similares, por lo cual podemos concluir que cualquiera de los filtros presentados puede ser utilizado para la digitalización de objetos, sin embargo se sugiere utilizar el filtro por umbral ya que con este filtro se obtuvieron la menor cantidad de inconsistencias.

\section{Referencias}

1. Takeda, M., Hideki, I., Seiji, K.: Fourier-transform method of fringe-pattern analysis for computer-based topography and interferometry. J. Opt. Soc. Am., 72, pp. 156-160 (1982) 
Comparación de filtros para el análisis del espectro espacial bidimensional de Fourier...

2. Takeda, M., Mutoh, K.: Fourier transform profilometry for the automatic measurement of 3-D object shapes. Appl. Opt., 22, pp. 3977-3982 (1983)

3. Jiang, Y., Shanglian, H.: Modified fourier transform profilometry for the measurement of 3-D steep shapes. Opfic.s und Laser.s in Engineerrng, 27, pp. 493-505 (1997)

4. Paulo, J., Tavares, M. A. V.: Orthogonal projection technique for resolution enhancement of the Fourier transform fringe analysis method. Optics Communications, 266, pp. 465-468 (2006)

5. Pedraza, J. C., Rodriguez, J. W., Barriga, L., Gorrostieta, E., Salgado, T., Ramos, J. M., Rivas, A.: Image Processing for 3D Reconstruction Using a Modified Fourier Transform Profilometry Method. Springer Berlin / Heidelberg, MICAI: Advances in Artificial Intelligence, 705-712 ISBN 3-540-76630-8 9783-540-76630-8 (2007)

6. Hussein, A. R.: Three-Dimensional Fourier Fringe Analysis and Phase Unwrapping. Tesis Doctoral General Engineering Research Institute (GERI), Liverpool John Moores University (2007)

7. Feng, S., Qian, C., Chao, Z., Jiasong, S., Tianyang, T.: A carrier removal technique for Fourier transform profilometry based on principal component analysis. Optics and Lasers in Engineering, 74, pp. 80-86 (2015)

8. Arevalillo, M., Gdeisat, M., Lilley, F., Burton, D.: A spatial algorithm to reduce phase wraps from two dimensional signals in fringe projection profilometry. Optics and Lasers in Engineering, 82, pp. 70-78 (2016)

9. Feng, L., Wenjing, C., Xianyu, S.: Eliminating zero spectra in Fourier transform profilometry by application of Hilbert transform. Optics Communications, 365, pp. 76-85 (2016)

10. Hussein, A. R., Gdeisat, M. A., Burton, D. R., Lalor, M. J., Lilley, F., Abdulbasit, A.: Three-dimensional Fourier Fringe Analysis. Optics and Lasers in Engineering, 46, ISSN: 0143-8166, pp. 446-455 (2008)

11. Guangliang, D., Chaorui, Z., Canlin, Z., Shuchun, S., Hui, L., Yanjie, L.: Iterative two-step temporal phase-unwrapping applied to high sensitivity threedimensional profilometry. Optics and Lasers in Engineering, 79, pp. 22-28 (2016)

12. Hai, L., Xin-yu, C., Fei, W., Xiao-Tang, H., Xiao-Dong, H.: A novel algorithm based on histogram processing of reliability for two-dimensional phase unwrapping. Optik, 126, pp. 1640-1644 (2015)

13. Mariano, R., Francisco, J. H., Adonai, G.: Phase unwrapping by accumulation of residual maps. Optics and Lasers in Engineering, 64, pp. 51-58 (2015)

14. Yuan, G., Xiaotian, C., Tao, Z.: Robust phase unwrapping algorithm based on least squares. Optics and Lasers in Engineering, 63, pp. 25-29 (2014) 